\title{
Nrf2 Is a Protective Factor against Oxidative Stresses Induced by Diesel Exhaust Particle in Allergic Asthma
}

\author{
Ying-Ji Li, ${ }^{1}$ Tomoyuki Kawada, ${ }^{1}$ and Arata Azuma ${ }^{2}$ \\ ${ }^{1}$ Department of Hygiene and Public Health, Graduate School of Medicine, Nippon Medical School, 1-1-5 Sendagi, Bunkyo-ku, \\ Tokyo 113-8602, Japan \\ ${ }^{2}$ Pulmonary Medicine/Infection and Oncology, Nippon Medical School, 1-1-5 Sendagi, Bunkyo-ku, Tokyo 113-8602, Japan
}

Correspondence should be addressed to Arata Azuma; azuma_arata@yahoo.co.jp

Received 11 January 2013; Revised 18 March 2013; Accepted 8 April 2013

Academic Editor: Hye-Youn Cho

Copyright (C) 2013 Ying-Ji Li et al. This is an open access article distributed under the Creative Commons Attribution License, which permits unrestricted use, distribution, and reproduction in any medium, provided the original work is properly cited.

\begin{abstract}
Epidemiological studies have shown that air pollutants, such as diesel exhaust particle (DEP), are implicated in the increased incidence of allergic airway disorders. In vitro studies of molecular mechanisms have focused on the role of reactive oxygen species generated directly and indirectly by the exposure to DEP. Antioxidants effectively reduce the allergic inflammatory effects induced by DEP both in vitro and in vivo. On the other hand, Nrf2 is a transcription factor essential for the inducible and/or constitutive expression of phase II and antioxidant enzymes. Disruption of Nrf2 enhances susceptibility to airway inflammatory responses and exacerbation of allergic inflammation induced by DEP in mice. Host responses to DEP are regulated by a balance between antioxidants and proinflammatory responses. Nrf2 may be an important protective factor against oxidative stresses induced by DEP in airway inflammation and allergic asthma and is expected to contribute to chemoprevention against DEP health effects in susceptible individuals.
\end{abstract}

\section{Introduction}

Air pollution is a complex mixture of compounds in gaseous and particle phases and the strongest evidence from many epidemiological studies linking air pollution with human health effects [1-4] centers around the particulate components. Particles are classified according to their aerodynamic diameter into size fractions such as particulate matter (PM) 10 (thoracic particles, $\leq 10 \mu \mathrm{m}$ ), PM2.5 (fine particulate matter of diameter, $\leq 2.5 \mu \mathrm{m}$ ), and ultrafine particles (UFP, fine particulate matter of diameter $\leq 0.1 \mu \mathrm{m}$ ) [5]. Many epidemiologic studies suggest that PM2.5 is associated with increased respiratory morbidity and mortality [6-8]. Particulate matter includes primary particles that are emitted directly from sources such as fossil-fuel combustion, for example, diesel exhaust particle (DEP), and secondary particles that are generated from gases through chemical reactions [5].
Air pollution from motor vehicles has been implicated as an important factor responsible for the increased prevalence of allergic diseases [9]. There is epidemiologic evidence of the impact of diesel exhaust or "near roadway" effects on asthma in humans, particularly children [4]. DEPs are the major components of ambient PM2.5 [10], especially in urban areas, and many in vivo and in vitro studies have been performed to clarify the association between DEP and pulmonary disorders such as asthma [11-15]. In vitro studies have shown that most of the effects of DEP were due to reactive oxygen species (ROS) generated by exposure to DEPs and the subsequent generation of the oxidative stress response within exposed cells [16-21].

A nuclear factor, erythroid-derived 2-like 2 (Nfe2l2) or NF-E2-related factor 2 (Nrf2), is a redox-sensitive basic leucine zipper transcription factor that is involved in the transcriptional regulation of many antioxidant genes [22] 
Extensive studies have suggested that Nrf2 contributes to protection against various pathologies, including asthma [2325], chronic obstructive pulmonary disease (COPD) [26], lung fibrosis [27], carcinogenesis [28], atherosclerosis [29], inflammatory disorders [30], and environmental oxidants, including hyperoxia [31, 32] and cigarette smoke [33]. Furthermore, it is implicated that Nrf2 subsidizes host defense through modulation of complex pathways, including wellcharacterized antioxidant activation in airway inflammation and allergic asthma induced by DEP [21]. A study of a small number of patients with allergic rhinitis showed that susceptibility to the adverse risks induced by DEP is partially dependent on glutathione-S-transferases (GST) genotypes in humans [34]. Oxidant stress seems to be involved in diesel-associated increases in airway inflammation [35] and allergic asthma [36], as evidenced by experiments in Nrf2 knockout mice. The present review describes recent research demonstrating the effect of $\mathrm{Nrf} 2$ in allergic asthma implicated in DEP exposure.

\section{Characteristics of Diesel Exhaust}

DEP produced by diesel engines is a major component of particulate atmospheric pollution, especially in urban areas. DEP has a complex structure characterized by a carbonaceous core with adsorbed organic compounds such as polyaromatic hydrocarbons (PAHs) and quinones. Their small size (0.1$0.3 \mu \mathrm{m}$ ) allows them to penetrate deeply into the respiratory tract and reach the pulmonary alveoli [37]. The PAHs and their oxygenated derivatives (e.g., quinones) have attracted special attention because they are able to participate in the redox cycle and generate ROS in target cells [38]. The main effect observed in healthy human volunteers exposed to DEP is inflammation characterized by an increase of inflammatory cells and chemokines and immunoglobulin E levels in nose lavages [13], which could account for the epidemiological association between chronic exposure to particulate matter and the increase of allergic diseases such as asthma and rhinitis [37].

\section{Nrf2 as a Key Regulator of Phase II Detoxifying Enzyme Genes and Antioxidant-Responsive Genes}

Nrf2 was discovered as a ubiquitous transcriptional regulator of antioxidant and detoxification genes. Nrf2 is a transcription factor essential for the inducible and/or constitutive expression of phase II and antioxidant enzymes. For instance, several GST isoforms and $\mathrm{NAD}(\mathrm{P}) \mathrm{H}$ :quinone oxidoreductase 1 (NQO1) were found to be uninducible by xenobiotics in the Nrf2 germ line mutant mouse [22]. These findings showed that Nrf2 has a major role in transcriptional activation through antioxidant responsive elements.

The expression of phase II detoxifying enzyme genes is clearly induced in wild-type and heterozygous Nrf2knockout mice, but the inducible expression of these genes is markedly reduced in homozygous Nrf2-knockout mice [39]. Insufficient induction of cytoprotective enzyme genes causes an increased susceptibility to various xenobiotics $[40,41]$ and components such as DEP $[35,42]$.

\section{DEP Induces ROS In Vitro and In Vivo, and Subsequent Nrf2 Activation Leads to Antioxidant Gene Expression}

Studies of molecular mechanisms have focused on the role of ROS generated directly and indirectly by exposure to DEP. ROS play an important role in proinflammatory reactions in airways. Enhanced inflammation involving the activation of alveolar macrophages following DEP exposure leads to the generation of ROS indirectly [43]; however, it is reported that intratracheal exposure to DEP caused the formation of 8-hydroxydeoxyguanosine in the murine lung [44]. DEP chemicals [45] and metals [46] could directly produce ROS such as superoxide and hydroxyl radical. These observations suggest that DEP can generate ROS, leading to oxidative stress-dependent pulmonary damage. DEP induce inflammatory cytokines such as interleukin- (IL-) 8 expression mediated by nuclear factor- (NF-) $\kappa \mathrm{B}$ in vitro, and these effects are blocked by antioxidant agents such as $\mathrm{N}$-acetyl cysteine (NAC) $[16,18]$. These observations also suggest that DEP-induced activation of signal pathways and transcription factors is due to ROS derived primarily from DEP.

In vitro research suggests that cytoprotective pathways are induced by the Nrf2 transcription signal pathway at the lowest levels of oxidative stress from DEP and can induce the transcription of antioxidant genes in the earliest level of defense. This may constitute the first tier of a hierarchical oxidative stress response. If these enzymes fail to neutralize the effects of ROS, proinflammatory effects constitute a second tier or superimposed level of oxidative stress. The final tier or superimposed level of oxidative stress is cytotoxicity, including the initiation of programmed cell death [19]. Nrf2 regulates antioxidant defense that is constituted as a main defense action against the proinflammatory and oxidizing effects of DEP [21].

In vivo studies with low-level and repeated DEP exposure $\left(100 \mu \mathrm{g} / \mathrm{m}^{3}\right)$ showed that DEP exposure induced airway inflammation in mice [47-50]. Host responses to DEP are regulated by a balance between antioxidant defenses and proinflammatory responses [38]. Studies of two different strains in mice demonstrated that there was a susceptibility difference to DEP exposure, and that certain antioxidant enzymes could be candidates as susceptibility genes $[49,50]$. DNA adduct formation has been shown to be accelerated in the lungs of Nrf2 knockout mice exposed to DEP [42]. Nrf2 knockout mice exposed to low-dose DEP for 8 weeks showed significantly increased airway hyperresponsiveness and counts of lymphocytes and eosinophils, together with increased concentrations of IL-12 and IL-13, and thymus and activation-regulated chemokine (TARC) in bronchoalveolar lavage (BAL) fluid compared with wild-type mice. In contrast, the expression of antioxidant enzyme genes was significantly higher in wild-type mice than in Nrf2 knockout mice [35]. This study strongly suggested that DEP-induced 
oxidative stress and host antioxidant responses were regulated by $\mathrm{Nrf} 2$.

It is known that DEP induces and exaggerates allergic airway inflammation in vivo $[13,51,52]$. Studies of a murine model of asthma, where mice received repeated low-level exposure to DEP, showed that DEP induced and exaggerated allergic airway inflammation, and NAC treatment reduced these allergic inflammatory responses caused by DEP [53]. Nrf2 knockout mice exposed to low-dose DEP showed significantly increased airway hyperresponsiveness and counts of lymphocytes, neutrophils, and eosinophils, together with increased concentrations of TARC in BAL fluid compared to wild-type mice in an asthma model [36]. TARC is a pivotal chemokine for the development of Th2-dominated experimental allergen-induced asthma with eosinophilia and airway hyperresponsiveness [54]. Increased inflammatory cells and PAS staining-positive mucus cell hyperplasia were evident in Nrf2 knockout mice. In contrast, the expression of GSH/GSSG (reduced glutathione/oxidized glutathione) was higher in wild-type mice than in Nrf2 knockout mice [36]. These results highlighted the role of DEP-induced oxidative stress and host antioxidant responses in the exaggeration of allergic airway inflammation in mice. It has been reported that the responsiveness of the Nrf2-directed antioxidant pathway acts as a major determinant of susceptibility to allergen-mediated asthma [23-25]. These reports suggest that the synergistic effects of oxidative stress caused by DEP and allergens contribute to the major pathway of the exaggeration of allergic asthma.

Disruption of Nrf2 enhances susceptibility to allergic airway inflammatory responses induced by low-dose DEP $\left(100 \mu \mathrm{g} / \mathrm{m}^{3}\right)$ in mice, but the data did not show any adjuvant activity of DEP for IgE production [36]. The effect of oxidative stresses caused by DEP may be crucial for the induction and exaggeration of allergic airway inflammatory responses due to DEP exposure in vivo.

\section{Future Direction: Nrf2 Is a Key Factor As a Potential Target of Chemoprevention}

The production of ROS was closely implicated in airway inflammation, allergy, and asthma; therefore, antioxidants may become a prophylactic strategy against adverse health effects of DEP. Chemoprevention by antioxidants has been reported to reduce the allergic inflammatory effects of DEP in mice [55]. NAC is widely known as an antioxidant drug. DEP-induced oxidants stress and the resultant inflammatory changes were blocked by NAC in asthma model [53]. Sulforaphane, a compound found in broccoli sprouts and broccoli, is also known to be a potent Nrf2 activator and is capable of preventing the toxicity of organic chemicals $[56,57]$. Sulforaphane-stimulated phase II enzyme induction inhibits cytokine production by airway epithelial cells stimulated with DEP [58].

Observations from these studies highlight the importance of the Nrf2-antioxidant pathway and may provide new therapeutic strategies for acute respiratory distress syndrome implicated in oxidative stress from DEP exposure.

\section{Conclusion}

Epidemiological, human, and animal experimental studies together suggest that DEP is involved in the recent increased prevalence of allergic diseases. Studies of molecular mechanisms have focused on the role of ROS generated directly and indirectly by exposure to diesel exhaust. Chemoprevention against DEP health effects in susceptible individuals may become a choice for a future environmental protection policy, and Nrf2 is a key potential target of chemoprevention.

\section{Acknowledgment}

This work was supported by the staff of the Department of Hygiene and Public Health, Nippon Medical School.

\section{References}

[1] Z. Xia, X. Duan, S. Tao et al., "Pollution level, inhalation exposure and lung cancer risk of ambient atmospheric polycyclic aromatic hydrocarbons (PAHs) in Taiyuan, China," Environmental Pollution, vol. 173, pp. 150-156, 2013.

[2] R. M. Krishnan, S. D. Adar, A. A. Szpiro et al., "Vascular responses to long- and short-term exposure to fine particulate matter: MESA Air (Multi-Ethnic Study of Atherosclerosis and Air Pollution)," Journal of the American College of Cardiology, vol. 60, no. 21, pp. 2158-2166, 2012.

[3] H. E. Volk, F. Lurmann, B. Penfold, I. Hertz-Picciotto, and R. McConnell, "Traffic-related air pollution, particulate matter, and autism," JAMA Psychiatry, vol. 70, no. 1, pp. 71-77, 2013.

[4] U. Gehring, A. H. Wijga, M. Brauer et al., "Traffic-related air pollution and the development of asthma and allergies during the first 8 years of life," American Journal of Respiratory and Critical Care Medicine, vol. 181, no. 6, pp. 596-603, 2010.

[5] J. A. Araujo and A. E. Nel, "Particulate matter and atherosclerosis: role of particle size, composition and oxidative stress," Particle and Fibre Toxicology, vol. 6, no. 24, pp. 1-19, 2009.

[6] D. W. Dockery, C. A. Pope III, X. Xu et al., "An association between air pollution and mortality in six U.S. cities," The New England Journal of Medicine, vol. 329, no. 24, pp. 1753-1759, 1993.

[7] J. M. Samet, F. Dominici, F. C. Curriero, I. Coursac, and S. L. Zeger, "Fine particulate air pollution and mortality in 20 U.S. cities, 1987-1994," The New England Journal of Medicine, vol. 343 , no. 24, pp. 1742-1749, 2000.

[8] G. Hoek, B. Brunekreef, S. Goldbohm, P. Fischer, and P. A. van den Brandt, "Association between mortality and indicators of traffic-related air pollution in the Netherlands: a cohort study," The Lancet, vol. 360, no. 9341, pp. 1203-1209, 2002.

[9] D. Diaz-Sanchez, L. Proietti, and R. Polosa, "Diesel fumes and the rising prevalence of atopy: an urban legend?" Current Allergy and Asthma Reports, vol. 3, no. 2, pp. 146-152, 2003.

[10] M. Sahu, S. Hu, P. H. Ryan et al., "Chemical compositions and source identification of PM2.5 aerosols for estimation of a diesel source surrogate," The Science of the Total Environment, vol. 409, no. 13, pp. 2642-2651, 2011.

[11] A. Saxon and D. Diaz-Sanchez, "Air pollution and allergy: you are what you breathe," Nature Immunology, vol. 6, no. 3, pp. 223226, 2005.

[12] D. Diaz-Sanchez, "The role of diesel exhaust particles and their associated polyaromatic hydrocarbons in the induction of 
allergic airway disease," Allergy, vol. 52, no. 38, supplement, pp. 52-56, 1997.

[13] D. Diaz-Sanchez, A. R. Dotson, H. Takenaka, and A. Saxon, "Diesel exhaust particles induce local IgE production in vivo and alter the pattern of IgE messenger RNA isoforms," Journal of Clinical Investigation, vol. 94, no. 4, pp. 1417-1425, 1994.

[14] S. Salvi, A. Blomberg, B. Rudell et al., "Acute inflammatory responses in the airways and peripheral blood after shortterm exposure to diesel exhaust in healthy human volunteers," American Journal of Respiratory and Critical Care Medicine, vol. 159, no. 3, pp. 702-709, 1999.

[15] N. Stenfors, C. Nordenhäll, S. S. Salvi et al., "Different airway inflammatory responses in asthmatic and healthy humans exposed to diesel," European Respiratory Journal, vol. 23, no. 1, pp. 82-86, 2004.

[16] H. Takizawa, T. Ohtoshi, S. Kawasaki et al., "Diesel exhaust particles induce NF- $\kappa$ B activation in human bronchial epithelial cells in vitro: importance in cytokine transcription," Journal of Immunology, vol. 162, no. 8, pp. 4705-4711, 1999.

[17] H. Takizawa, S. Abe, H. Okazaki et al., "Diesel exhaust particles upregulate eotaxin gene expression in human bronchial epithelial cells via nuclear factor- $\kappa \mathrm{B}$-dependent pathway," American Journal of Physiology, vol. 284, no. 6, pp. L1055-L1062, 2003.

[18] S. Hashimoto, Y. Gon, I. Takeshita et al., "Diesel exhaust particles activate p38 MAP kinase to produce interleukin 8 and RANTES by human bronchial epithelial cells and Nacetylcysteine attenuates p38 MAP kinase activation," American Journal of Respiratory and Critical Care Medicine, vol. 161, no. 1, pp. 280-285, 2000.

[19] G. G. Xiao, M. Wang, N. Li, J. A. Loo, and A. E. Nel, "Use of proteomics to demonstrate a hierarchical oxidative stress response to diesel exhaust particle chemicals in a macrophage cell line," Journal of Biological Chemistry, vol. 278, no. 50, pp. 50781-50790, 2003.

[20] N. Li, M. I. Venkatesan, A. Miguel et al., "Induction of heme oxygenase-1 expression in macrophages by diesel exhaust particle chemicals and quinones via the antioxidant-responsive element," Journal of Immunology, vol. 165, no. 6, pp. 3393-3401, 2000.

[21] N. Li, J. Alam, M. I. Venkatesan et al., "Nrf2 is a key transcription factor that regulates antioxidant defense in macrophages and epithelial cells: protecting against the proinflammatory and oxidizing effects of diesel exhaust chemicals," Journal of Immunology, vol. 173, no. 5, pp. 3467-3481, 2004.

[22] K. Itoh, T. Chiba, S. Takahashi et al., "An Nrf2/small Maf heterodimer mediates the induction of phase II detoxifying enzyme genes through antioxidant response elements," Biochemical and Biophysical Research Communications, vol. 236, no. 2, pp. 313-322, 1997.

[23] T. Rangasamy, J. Guo, W. A. Mitzner et al., "Disruption of Nrf2 enhances susceptibility to severe airway inflammation and asthma in mice," Journal of Experimental Medicine, vol. 202, no. 1, pp. 47-59, 2005.

[24] C. Michaeloudes, P. J. Chang, M. Petrou, and K. F. Chung, "Transforming growth factor- $\beta$ and nuclear factor E2-related factor 2 regulate antioxidant responses in airway smooth muscle cells: role in asthma," American Journal of Respiratory and Critical Care Medicine, vol. 184, no. 8, pp. 894-903, 2011.

[25] A. M. Fitzpatrick, S. T. Stephenson, G. R. Hadley et al., “Thiol redox disturbances in children with severe asthma are associated with posttranslational modification of the transcription factor nuclear factor (erythroid-derived 2)-like 2," Journal of
Allergy and Clinical Immunology, vol. 127, no. 6, pp. 1604-1611, 2011.

[26] D. Malhotra, R. K. Thimmulappa, N. Mercado et al., "Denitrosylation of HDAC2 by targeting Nrf2 restores glucocorticosteroid sensitivity in macrophages from COPD patients," The Journal of Clinical Investigation, vol. 121, no. 11, pp. 4289-4302, 2011.

[27] H. Y. Cho, S. P. M. Reddy, M. Yamamoto, and S. R. Kleeberger, "The transcription factor NRF2 protects against pulmonary fibrosis," FASEB Journal, vol. 18, no. 11, pp. 1258-1260, 2004.

[28] A. Ohkoshi, T. Suzuki, M. Ono, T. Kobayashi, and M. Yamamoto, "Roles of Keap1-Nrf2 system in upper aerodigestive tract carcinogenesis," Cancer Prevention Research, vol. 6, no. 2, pp. 149-159, 2013.

[29] T. E. Sussan, J. Jun, R. Thimmulappa et al., "Disruption of Nrf2, a key inducer of antioxidant defenses, attenuates ApoE-mediated atherosclerosis in mice," PLoS One, vol. 3, no. 11, Article ID e3791, 2008.

[30] J. Kim, Y. N. Cha, and Y. J. Surh, "A protective role of nuclear factor-erythroid 2-related factor-2 (Nrf2) in inflammatory disorders," Mutation Research, vol. 690, no. 1-2, pp. 12-23, 2010.

[31] H. Y. Cho, A. E. Jedlicka, S. P. M. Reddy, L. Y. Zhang, T. W. Kensler, and S. R. Kleeberger, "Linkage analysis of susceptibility to hyperoxia Nrf2 is a candidate gene," American Journal of Respiratory Cell and Molecular Biology, vol. 26, no. 1, pp. 42-51, 2002.

[32] H. Y. Cho, A. E. Jedlicka, S. P. Reddy et al., "Role of NRF2 in protection against hyperoxic lung injury in mice," American Journal of Respiratory Cell and Molecular Biology, vol. 26, no. 2, pp. 175-182, 2002.

[33] M. Suzuki, T. Betsuyaku, Y. Ito et al., "Down-regulated NF-E2related factor 2 in pulmonary macrophages of aged smokers and patients with chronic obstructive pulmonary disease," American Journal of Respiratory Cell and Molecular Biology, vol. 39, no. 6, pp. 673-682, 2008.

[34] F. D. Gilliland, Y. F. Li, A. Saxon, and D. Diaz-Sanchez, "Effect of glutathione-S-transferase M1 and P1 genotypes on xenobiotic enhancement of allergic responses: randomised, placebo-controlled crossover study," The Lancet, vol. 363, no. 9403, pp. 119-125, 2004.

[35] Y. J. Li, H. Takizawa, A. Azuma et al., "Disruption of Nrf2 enhances susceptibility to airway inflammatory responses induced by low-dose diesel exhaust particles in mice," Clinical Immunology, vol. 128, no. 3, pp. 366-373, 2008.

[36] Y. J. Li, H. Takizawa, A. Azuma et al., "Nrf2 is closely related to allergic airway inflammatory responses induced by low-dose diesel exhaust particles in mice," Clinical Immunology, vol. 137, no. 2, pp. 234-241, 2010.

[37] A. Baulig, M. Garlatti, V. Bonvallot et al., "Involvement of reactive oxygen species in the metabolic pathways triggered by diesel exhaust particles in human airway epithelial cells," American Journal of Physiology, vol. 285, no. 3, pp. L671-L679, 2003.

[38] H. Takizawa, "Diesel exhaust particles and their effect on induced cytokine expression in human bronchial epithelial cells," Current Opinion in Allergy and Clinical Immunology, vol. 4, no. 5, pp. 355-359, 2004.

[39] J. D. Hayes, S. A. Chanas, C. J. Henderson et al., "The Nrf2 transcription factor contributes both to the basal expression of glutathione S-transferases in mouse liver and to their induction by the chemopreventive synthetic antioxidants, butylated hydroxyanisole and ethoxyquin," Biochemical Society Transactions, vol. 28, no. 2, pp. 33-41, 2000. 
[40] K. Chan and Y. W. Kan, "Nrf2 is essential for protection against acute pulmonary injury in mice," Proceedings of the National Academy of Sciences of the United States of America, vol. 96, no. 22, pp. 12731-12736, 2000.

[41] A. Enomoto, K. Itoh, E. Nagayoshi et al., "High sensitivity of Nrf2 knockout mice to acetaminophen hepatotoxicity associated with decreased expression of ARE-regulated drug metabolizing enzymes and antioxidant genes," Toxicological Sciences, vol. 59, no. 1, pp. 169-177, 2001.

[42] Y. Aoki, H. Sato, N. Nishimura, S. Takahashi, K. Itoh, and M. Yamamoto, "Accelerated DNA adduct formation in the lung of the Nrf2 knockout mouse exposed to diesel exhaust," Toxicology and Applied Pharmacology, vol. 173, no. 3, pp. 154-160, 2001.

[43] A. M. Casillas, T. Hiura, N. Li, and A. E. Nel, "Enhancement of allergic inflammation by diesel exhaust particles: permissive role of reactive oxygen species," Annals of Allergy, Asthma and Immunology, vol. 83, no. 6, pp. 624-629, 1999.

[44] M. Nagashima, H. Kasai, J. Yokota, Y. Nagamachi, T. Ichinose, and M. Sagai, "Formation of an oxidative DNA damage, 8hydroxydeoxyguanosine, in mouse lung DNA after intratracheal instillation of diesel exhaust particles and effects of high dietary fat and beta-carotene on this process," Carcinogenesis, vol. 16, no. 6, pp. 1441-1445, 1995.

[45] M. Sagai, H. Saito, T. Ichinose, M. Kodama, and Y. Mori, "Biological effects of diesel exhaust particles. I. In vitro production of superoxide and in vivo toxicity in mouse," Free Radical Biology and Medicine, vol. 14, no. 1, pp. 37-47, 1993.

[46] A. K. Prahalad, J. M. Soukup, J. Inmon et al., "Ambient air particles: effects on cellular oxidant radical generation in relation to particulate elemental chemistry," Toxicology and Applied Pharmacology, vol. 158, no. 2, pp. 81-91, 1999.

[47] Y. Saito, A. Azuma, S. Kudo, H. Takizawa, and I. Sugawara, "Long-term inhalation of diesel exhaust affects cytokine expression in murine lung tissues: comparison between low- and highdose diesel exhaust exposure," Experimental Lung Research, vol. 28, no. 6, pp. 493-506, 2002.

[48] K. Hiramatsu, A. Azuma, S. Kudoh, M. Desaki, H. Takizawa, and I. Sugawara, "Inhalation of diesel exhaust for three months affects major cytokine expression and induces bronchusassociated lymphoid tissue formation in murine lungs," Experimental Lung Research, vol. 29, no. 8, pp. 607-622, 2003.

[49] Y. J. Li, A. Matsumoto, H. Takizawa et al., "Airway inflammatory responses to oxidative stress induced by low-dose diesel exhaust particle exposure differ between mouse strains," Experimental Lung Research, vol. 33, no. 5, pp. 227-244, 2007.

[50] Y. J. Li, T. Kawada, H. Takizawa et al., "Airway inflammatory responses to oxidative stress induced by prolonged low-dose diesel exhaust particle exposure from birth differ between mouse BALB/c and C57BL/6 strains," Experimental Lung Research, vol. 34, no. 3, pp. 125-139, 2008.

[51] H. Takano, T. Yoshikawa, T. Ichinose, Y. Miyabara, K. Imaoka, and M. Sagai, "Diesel exhaust particles enhance antigeninduced airway inflammation and local cytokine expression in mice," American Journal of Respiratory and Critical Care Medicine, vol. 156, no. 1, pp. 36-42, 1997.

[52] Y. Miyabara, H. Takano, T. Ichinose, H. B. Lim, and M. Sagai, "Diesel exhaust enhances allergic airway inflammation and hyperresponsiveness in mice," American Journal of Respiratory and Critical Care Medicine, vol. 157, no. 4, pp. 1138-1144, 1998.

[53] Y. J. Li, H. Takizawa, A. Azuma et al., "The effects of oxidative stress induced by prolonged low-dose diesel exhaust particle exposure on the generation of allergic airway inflammation differ between BALB/c and C57BL/6 mice," Immunopharmacology and Immunotoxicology, vol. 31, no. 2, pp. 230-237, 2009.

[54] S. Kawasaki, H. Takizawa, H. Yoneyama et al., "Intervention of thymus and activation-regulated chemokine attenuates the development of allergic airway inflammation and hyperresponsiveness in mice," Journal of Immunology, vol. 166, no. 3, pp. 2055-2062, 2001.

[55] M. J. Whitekus, N. Li, M. Zhang et al., "Thiol antioxidants inhibit the adjuvant effects of aerosolized diesel exhaust particles in a murine model for ovalbumin sensitization," Journal of Immunology, vol. 168, no. 5, pp. 2560-2567, 2002.

[56] J. W. Fahey, X. Haristoy, P. M. Dolan et al., "Sulforaphane inhibits extracellular, intracellular, and antibioticresistant antibioticresistant strains of Helicobacter pylori and prevents benzo[a]pyrene-induced stomach tumors," Proceedings of the National Academy of Sciences of the United States of America, vol. 99, no. 11, pp. 7610-7615, 2002.

[57] K. Iida, K. Itoh, Y. Kumagai et al., "Nrf2 is essential for the chemopreventive efficacy of oltipraz against urinary bladder carcinogenesis," Cancer Research, vol. 64, no. 18, pp. 6424-6431, 2004.

[58] S. A. Ritz, J. Wan, and D. Diaz-Sanchez, "Sulforaphanestimulated phase II enzyme induction inhibits cytokine production by airway epithelial cells stimulated with diesel extract," American Journal of Physiology, vol. 292, no. 1, pp. L33-L39, 2007. 


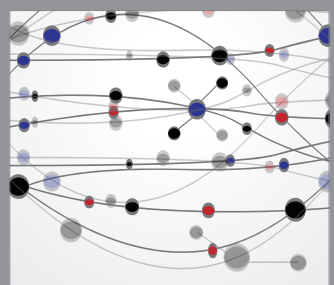

The Scientific World Journal




Gastroenterology

Research and Practice
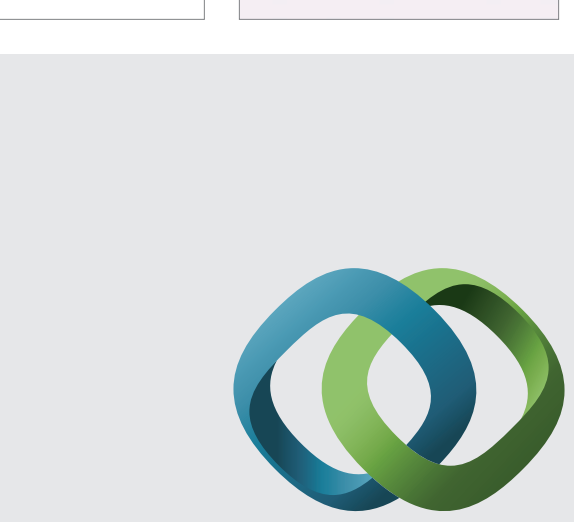

\section{Hindawi}

Submit your manuscripts at

http://www.hindawi.com
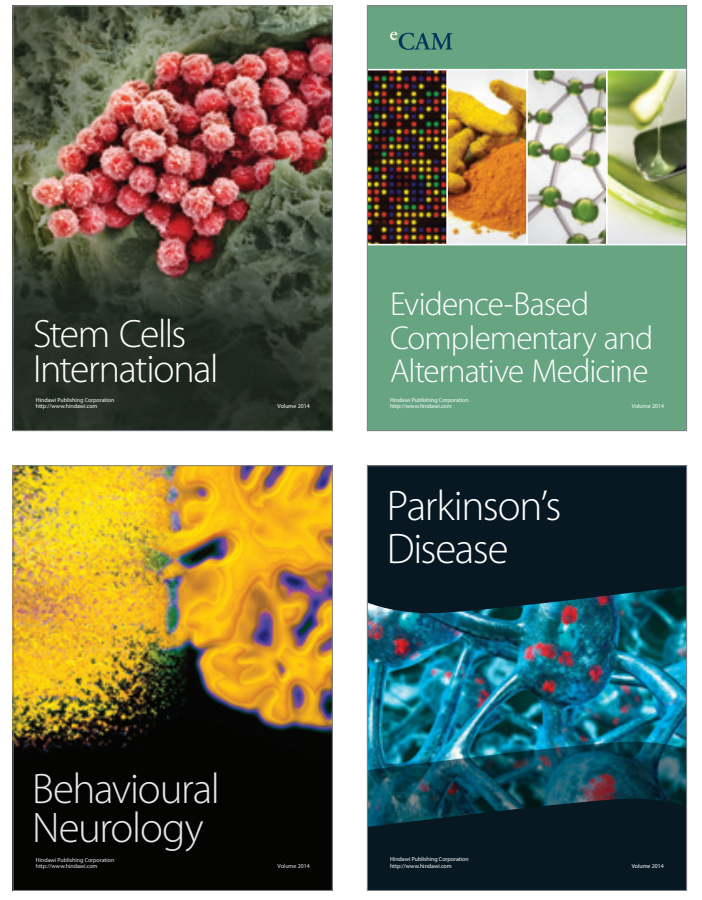


Disease Markers
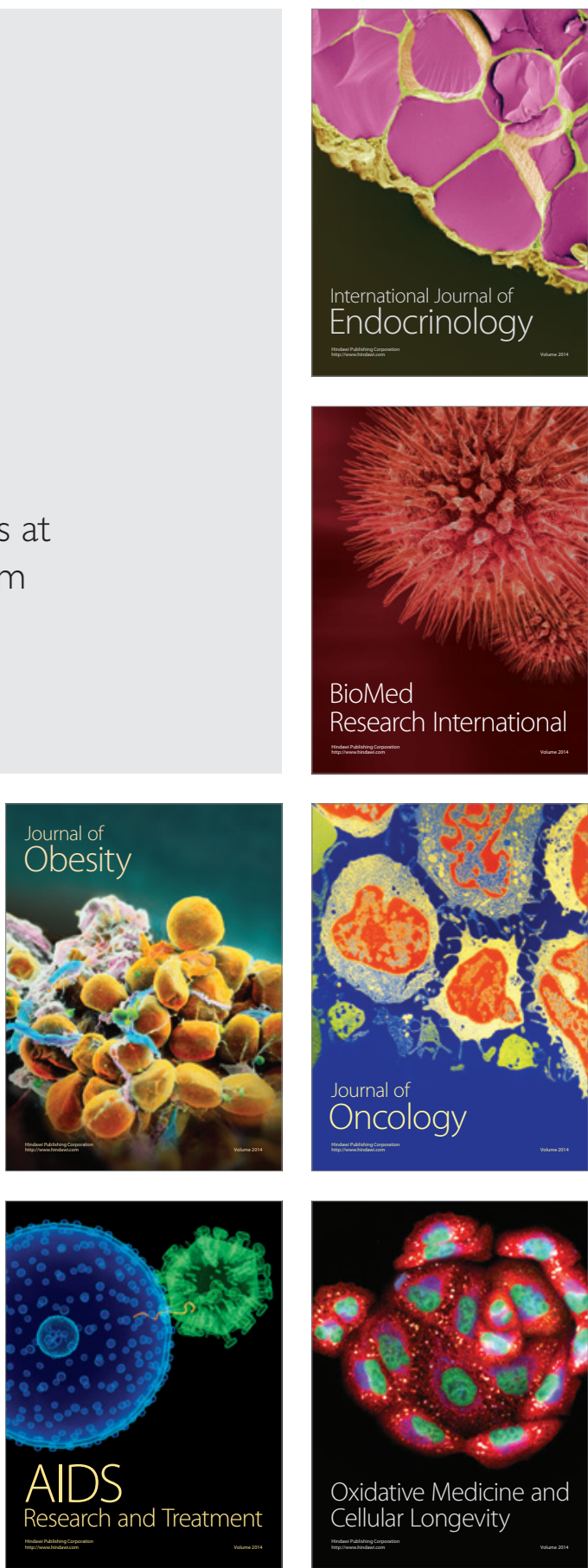